\title{
Fifth-Order Finite-Volume WENO on Cylindrical Grids
}

\author{
Mohammad Afzal Shadab, Xing Ji, and Kun Xu
}

\section{Introduction}

The conventional WENO scheme is specifically designed for the reconstruction in Cartesian coordinates on uniform grids [1]. The employment of Cartesian-based reconstruction scheme on a cylindrical grid suffers from a number of drawbacks $[2,3]$, e.g., in the original PPM paper, reconstruction was performed in volume coordinates (than the linear ones) so that algorithm for a Cartesian mesh can be used on a curvilinear mesh. However, the resulting interface states became first-order accurate even for smooth flows [2]. Another example can be the volume average assignment to the geometrical cell center of finite-volume than the centroid [2]. A breakthrough in the field of high order reconstruction in cylindrical coordinates is the application of the Vandermonde-like linear systems of equations with spatially

\footnotetext{
M. A. Shadab $(\bowtie)$

Department of Mechanical and Aerospace Engineering, Hong Kong University of Science and Technology, Kowloon City, Hong Kong

X. Ji

Department of Mathematics, Hong Kong University of Science and Technology, Kowloon City, Hong Kong

e-mail:xjiad@connect.ust.hk

K. Xu

Department of Mathematics, Hong Kong University of Science and Technology, Kowloon City, Hong Kong

Department of Mechanical and Aerospace Engineering, Hong Kong University of Science and Technology, Kowloon City, Hong Kong

e-mail: makxu@ust.hk

(C) The Author(s) 2020 
varying coefficients [2]. It is reintroduced in the present work to build a basis for the derivation of the high order WENO schemes.

The motivation for the present work is to develop a fifth-order finite-volume WENO reconstruction scheme in the efficient dimension-by-dimension framework, specifically aimed at regularly-spaced and irregularly-spaced grids in cylindrical coordinates.

\section{Finite-Volume Discretization in Curvilinear Coordinates}

\subsection{Evaluation of the Linear Weights}

A non-uniform grid spacing with zone width $\Delta \xi_{i}=\xi_{i+\frac{1}{2}}-\xi_{i-\frac{1}{2}}$ is considered having $\xi \in\left(x_{1}, x_{2}, x_{3}\right)$ as the coordinate along the reconstruction direction and $\xi_{i+\frac{1}{2}}$ denoting the location of the cell interface between zones $i$ and $i+1$. Let $\bar{Q}_{i}$ be the cell average of conserved quantity $Q$ inside zone $i$ at some given time, which can be expressed in form of Eq. (1).

$$
\bar{Q}_{i}=\frac{1}{\Delta \mathscr{V}_{i}} \int_{\xi_{i-\frac{1}{2}}}^{\xi_{i+\frac{1}{2}}} Q_{i}(\xi) \frac{\partial \mathscr{V}}{\partial \xi} d \xi \quad \& \quad \Delta \mathscr{V}_{i}=\int_{\xi_{i-\frac{1}{2}}}^{\xi_{i+\frac{1}{2}}} \frac{\partial \mathscr{V}}{\partial \xi} d \xi
$$

where the local cell volume $\Delta \mathscr{V}_{i}$ of $i$ th cell in the direction of reconstruction given in Eq. (1) and $\frac{\partial \mathscr{V}}{\partial \xi}$ is the one-dimensional Jacobian. Now, our aim is to find a $p$ th order accurate approximation to the actual solution by constructing a $(p-1)$ th order polynomial distribution, as given in Eq. (2).

$$
Q_{i}(\xi)=a_{i, 0}+a_{i, 1}\left(\xi-\xi_{i}^{c}\right)+a_{i, 2}\left(\xi-\xi_{i}^{c}\right)^{2}+\ldots+a_{i, p-1}\left(\xi-\xi_{i}^{c}\right)^{p-1}
$$

where $a_{i, n}$ corresponds to a vector of the coefficients which needs to be determined and $\xi_{i}^{c}$ can be taken as the cell centroid. However, the final values at the interface are independent of the particular choice of $\xi_{i}^{c}$ and one may as well set $\xi_{i}^{c}=0$ [2]. Unlike the cell center, the centroid is not equidistant from the cell interfaces in the case of cylindrical-radial coordinates, and the cell averaged values are assigned at the centroid [2]. Further, the method has to be locally conservative, i.e., the polynomial $Q_{i}(\xi)$ must fit the neighboring cell averages, satisfying Eq. (3).

$$
\int_{\xi_{i+s-\frac{1}{2}}}^{\xi_{i+s+\frac{1}{2}}} Q_{i}(\xi) \frac{\partial \mathscr{V}}{\partial \xi} d \xi=\Delta \mathscr{V}_{i+s} \bar{Q}_{i+s} \quad \text { for } \quad-i_{L} \leq s \leq i_{R}
$$

where the stencil includes $i_{L}$ cells to the left and $i_{R}$ cells to the right of the $i$ th zone such that $i_{L}+i_{R}+1=p$. Implementing Eqs. (1)-(2) in Eq. (3) along with a simple mathematical manipulation leads to Eq. (4), which is the fundamental equation for 
reconstruction in cylindrical coordinates. For the detailed derivation, kindly refer to [3].

$$
\left(\begin{array}{ccc}
\beta_{i-i_{L}, 0} & \ldots & \beta_{i-i_{L}, p-1} \\
\vdots & \ddots & \vdots \\
\beta_{i+i_{R}, 0} & \ldots & \beta_{i+i_{R}, p-1}
\end{array}\right)^{T}\left(\begin{array}{c}
w_{i,-i_{L}}^{ \pm} \\
\vdots \\
w_{i, i_{R}}^{ \pm}
\end{array}\right)=\left(\begin{array}{c}
1 \\
\vdots \\
\left(\xi_{i \pm \frac{1}{2}}-\xi_{i}^{c}\right)^{p-1}
\end{array}\right)
$$

where ' \pm ' represents the positive and negative weights i.e. weights for reconstructing right $(+)$ and left $(-)$ interface values respectively. Also, the grid dependent linear weights $\left(w_{i, s}^{ \pm}\right)$satisfy the normalization condition [2].

\subsection{Optimal Weights}

For the case of fifth-order WENO interpolation, the third order interpolated variables are optimally weighed in order to achieve fifth-order accurate interpolated values as given in Eq. (5) for the case of $p=3$ [1].

$$
q_{i, 0}^{(2 p-1) \pm}=\sum_{l=0}^{p-1} C_{i, l}^{ \pm} q_{i, l}^{p \pm}
$$

where $C_{i, l}^{ \pm}$is the optimal weight for the positive/negative cases on the $i$ th finitevolume. So, Eq. (4) is used again to evaluate the weights for the fifth-order $(2 p-1=$ $5)$ interpolation $\left(i_{L}=2, i_{R}=2\right)$.

Linear and optimal weights are independent of the mesh size for standard regularly-spaced grid cases. They can be evaluated and stored (at a nominal cost) independently before the actual computation. Also, they conform to the original WENO-JS [1] for the limiting case $(R \rightarrow \infty)$. The weights required for source term and flux integration in one or more dimensions are given in [3].

\subsection{Smoothness Indicators and the Nonlinear Weights}

The mathematical definition of the smoothness indicator is given in Eq. (6) [1].

$$
I S_{i, l}=\sum_{m=1}^{p-1} \int_{\xi_{j-\frac{1}{2}}}^{\xi_{j+\frac{1}{2}}}\left(\frac{d^{m}}{d \xi^{m}} Q_{i, l}(\xi)\right)^{2} \Delta \xi_{i}^{2 m-1} d \xi, \quad l=0, \ldots, p-1
$$

To evaluate the value of $I S_{i, l}$, a third order polynomial interpolation on $i$ th cell is required using positive and negative reconstructed values by stencil $S_{l}$, as given in Eq. (2). Finally, evaluating the values of the coefficient $a$ 's and substituting their 
values in smoothness indicator formula (6) yields the grid-independent fundamental relation (7). The nonlinear weight $\left(\omega_{i, l}^{ \pm}\right)$for the WENO-C interpolation is defined in Eq. (8) [1], where $\epsilon$ is chosen to be $10^{-6}[1,3]$.

$$
\begin{gathered}
I S_{i, l}=4\left(39 \bar{Q}_{i}^{2}-39 \bar{Q}_{i}\left(q_{i, l}^{-}+q_{i, l}^{+}\right)+10\left(\left(q_{i, l}^{-}\right)^{2}+\left(q_{i, l}^{+}\right)^{2}\right)+19 q_{i, l}^{-} q_{i, l}^{+}\right) \\
\omega_{i, l}^{ \pm}=\frac{\alpha_{i, l}^{ \pm}}{\sum_{l=0}^{p-1} \alpha_{i, l}^{ \pm}} \quad \& \quad \alpha_{i, l}^{ \pm}=\frac{C_{i, l}^{ \pm}}{\left(\epsilon+I S_{i, l}\right)^{2}} \quad l=0,1,2
\end{gathered}
$$

The final interpolated interface values are evaluated from Eq. (9).

$$
q_{i}^{(2 p-1) \pm}=\sum_{l=0}^{p-1} \omega_{i, l}^{p \pm} q_{i, l}^{p \pm}
$$

\section{Stability Analysis of WENO-C for Hyperbolic Conservation Laws}

For WENO-C to be practically useful, it is crucial that it enables a stable discretization for hyperbolic conservation laws when coupled with a proper time-integration scheme. In this section, we analyze WENO-C scheme for model problems involving smooth flow in 1D cylindrical-radial coordinates, based on a modified von Neumann stability analysis [4]. We consider scalar advection equation (10) in 1D cylindricalradial coordinates.

$$
\frac{\partial Q}{\partial t}+\frac{1}{(\partial \mathscr{V} / \partial \xi)} \frac{\partial}{\partial \xi}\left(\left(\frac{\partial \mathscr{V}}{\partial \xi}\right) Q v\right)=0 \quad \xi \in[0, \infty], \quad t>0
$$

where $Q$ is the conserved variable, $(\partial \mathscr{V} / \partial \xi)=\xi$ is the one-dimensional Jacobian in cylindrical-radial coordinates. Boundary conditions are not considered in the present approach to reduce the complexity of the analysis. Assuming a uniform grid with $\xi_{i}=i \Delta \xi$ and $\xi_{i+1}-\xi_{i}=\Delta \xi \forall i$ and $(i \pm 1 / 2)$ denotes the boundaries of the finitevolume $i$. In the finite-volume framework, Eq. (10) transforms into the conservative scheme given in Eq. (11).

$$
\frac{\partial \bar{Q}_{i}}{\partial t}=-\frac{1}{\Delta \mathscr{V}_{i}}\left(\hat{F}_{i+1 / 2}-\hat{F}_{i-1 / 2}\right)
$$

where numerical flux $\hat{F}_{i+1 / 2}$ is the Lax-Friedrich flux, and $\bar{Q}_{i}$ and $\mathscr{V}_{i}$ are given in Eq. (1). For this particular problem, let $v=1$ in Eq. (10). Therefore, only the values on the left side of the interface are considered. Based on the von Neumann stability analysis, the semi-discrete solution can be expressed as a discrete Fourier series. By the superposition principle, only one term in the series can be used for analysis, as 
illustrated in Eq. (12).

$$
\bar{Q}_{i}(t)=\hat{Q}_{k}(t) e^{j i \theta_{k}}, \quad \text { where } \quad j=\sqrt{-1}
$$

By substituting Eq. (12) in Eq. (11), we can separate the spatial operator $L$, as given in Eq. (13).

$$
L=-\frac{\left(\hat{F}_{i+1 / 2}-\hat{F}_{i-1 / 2}\right)}{\Delta \mathscr{V}_{i}}=-\frac{[Q(\partial \mathscr{V} / \partial \xi)]_{i+1 / 2}^{-}-[Q(\partial \mathscr{V} / \partial \xi)]_{i-1 / 2}^{-}}{\Delta \mathscr{V}_{i}}=-\frac{z\left(\theta_{k}\right) \bar{Q}_{i}}{\Delta \xi}
$$

where the complex function $z\left(\theta_{k}\right)$ is the Fourier symbol. By substituting the values of $Q_{i-1 / 2}^{-}$and $Q_{i+1 / 2}^{-}$using fifth-order positive weights of cells $(i-1)$ and $i$ respectively for a smooth solution, the value of $z\left(\theta_{k}\right)$ for WENO-C can be evaluated using Eq. (14).

$$
z\left(\theta_{k}\right)=\frac{m+1}{i^{(m+1)}-(i-1)^{(m+1)}} \sum_{l=-2}^{+2}\left[w_{i, l}^{+} i^{m} e^{j l \theta_{k}}-w_{(i-1), l}^{+}(i-1)^{m} e^{j(l-1) \theta_{k}}\right]
$$

where $m=1$ for cylindrical-radial coordinates. Using the same approach as given in [4], we can plot the spatial spectrum $\left\{S:-z\left(\theta_{k}\right)\right.$ for $\left.\theta_{k} \in[0,2 \pi]\right\}$ and the stability domain $S_{t}$ for TVD-RK order 3 . The maximum stable CFL number of this scheme can be computed by finding the largest rescaling parameter $\tilde{\sigma}$, so that the rescaled spectrum still lies in the stability domain.

It can be observed from Fig. 1 that the spatial spectrums $S$ of WENO-C differs initially with the index numbers $i$ due to the geometrical variation of the finitevolume. However, the spectrums are the same for high index numbers $(i)$, similar to WENO-JS, as the fifth-order interpolation weights converge. Some regions $(i=1,2)$ require boundary conditions and thus, are not considered in the present analysis. The values of CFL number for cylindrical-radial coordinates lie in between 1.45 and 1.52. As a final remark, it can be concluded that the proposed scheme is A-stable with third or higher order of RK method with an appropriate value of CFL number for this case.

\section{Numerical Tests}

In this section, several tests on Euler equations are performed to analyze the performance of the WENO-C reconstruction scheme. Tests are performed on a gamma law gas $(\gamma=1.4)$ in cylindrical coordinates to investigate the essentially non-oscillatory property of WENO-C for discontinuous flows and the convex combination property for smooth flows. For first-order and second-order (MUSCL) 

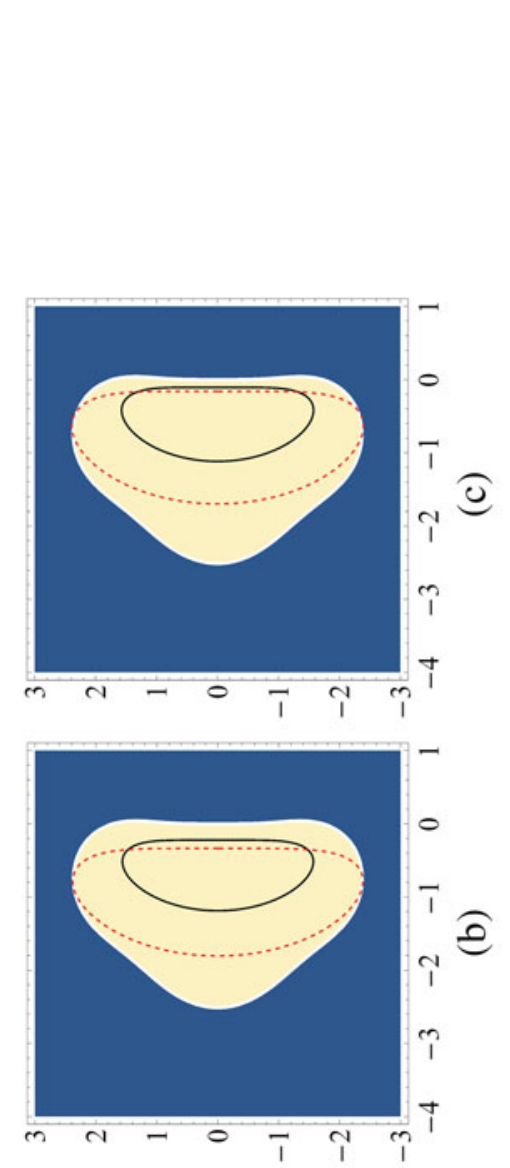

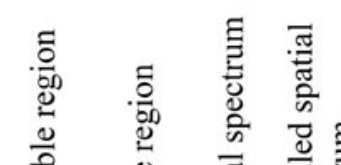

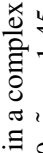

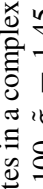

过

冚 $I$

อ

홍

픈

ป

E

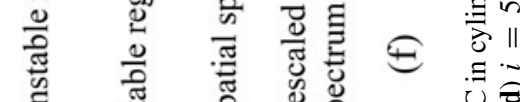

已ิ

Zิำ

3

可

i

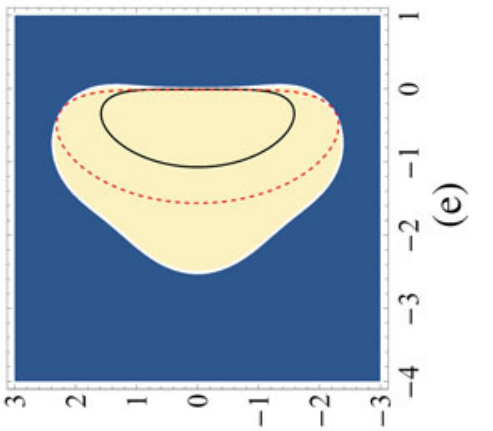

至

4

.

ถี กี่

는

需

‥

16 3

๖े
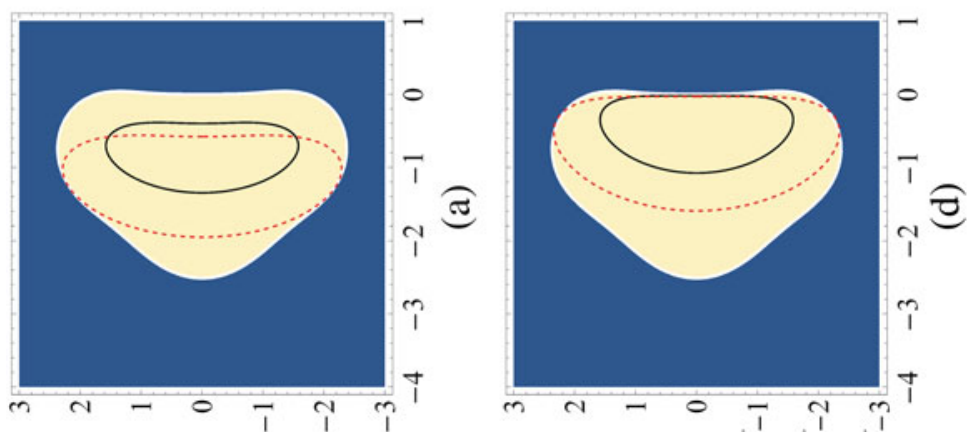

无

原 6

(1)

क्षิ.

छ

节.

気

芩

.气

3

है

可

क

过 
spatial reconstructions, Euler time marching and Maccormack (predictor-corrector) schemes are respectively employed. For WENO-C, time marching is done with TVD-RK order 3 for $1 \mathrm{D}$ cases and RK order 5 for the 2D case.

\subsection{Acoustic Wave Propagation}

A smooth problem involving a nonlinear system of 1D gas dynamical equations is solved to test fifth-order accuracy of the spatial discretization scheme [3]. The Euler equations in cylindrical-radial coordinates can be written in the form of Eq. (15).

$$
\frac{\partial}{\partial t}\left(\begin{array}{c}
\rho \\
\rho u \\
E
\end{array}\right)+\frac{1}{R} \frac{\partial}{\partial R}\left(\begin{array}{c}
\rho u R \\
\left(\rho u^{2}+p\right) R \\
(E+p) u R
\end{array}\right)=\left(\begin{array}{c}
0 \\
p / R \\
0
\end{array}\right)
$$

where $\rho$ is the mass density, $u$ is the radial velocity, $p$ is the pressure, and $E$ is the total energy. Equation (16) serves as the adiabatic equation of state.

$$
E=\frac{p}{\gamma-1}+\frac{1}{2} \rho u^{2}
$$

The initial conditions are provided in Eq. (17) with the perturbation given in Eq. (18). The interface flux is evaluated with Rusanov scheme [3].

$$
\begin{gathered}
\rho(R, 0)=1+\varepsilon f(R), \quad u(R, 0)=0, \quad p(R, 0)=1 / \gamma+\varepsilon f(R) \\
f(R)= \begin{cases}\frac{\sin ^{4}(5 \pi R)}{R} & \text { if } 0.4 \leq R \leq 0.6 \\
0 & \text { otherwise }\end{cases}
\end{gathered}
$$

A sufficiently small perturbation with $\varepsilon=10^{-4}$ yields a smooth solution. The interface flux is evaluated using Rusanov scheme with a CFL number of 0.3 .

The initial perturbation splits into two acoustic waves traveling in opposite directions. The final time $(t=0.3)$ is set such that the waves remain in the domain and the problem is free from the boundary effects. The computational domain of unity length is uniformly divided into $N$ different zones i.e. $N=$ $16,32,64,128,256$. Although an exact solution known up to $\mathrm{O}\left(\varepsilon^{2}\right)$ is known, the solution on the finest mesh $N=1024$ is taken as the reference. Figure 2 illustrate the spatial variation of density at $t=0.3$ inside the domain. From Table 1, it clear that the scheme approaches the desired fifth-order accuracy. 


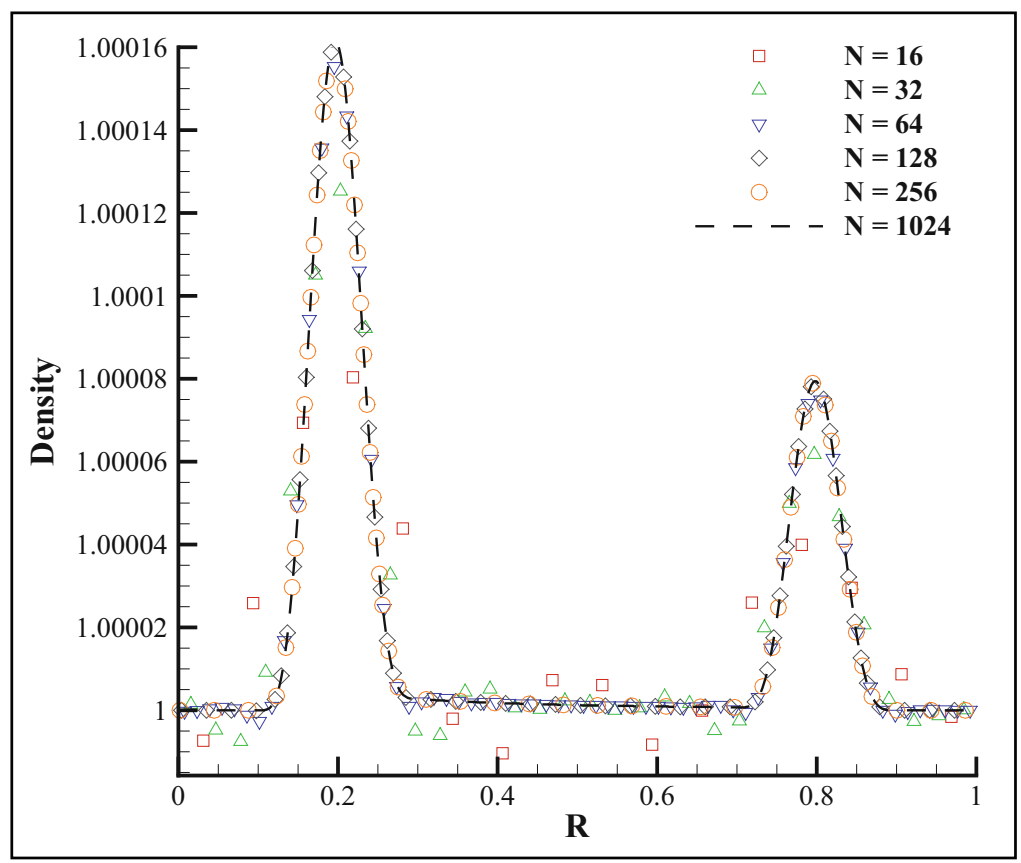

Fig. 2 Spatial profiles of density at $t=0.3$ for acoustic wave propagation test in cylindrical-radial coordinates

Table $1 L_{1}$ norm errors and order of convergence table for acoustic wave propagation test

\begin{tabular}{r|l|l}
\hline$N$ & $\epsilon(\rho)$ & $O_{L_{1}}$ \\
\hline 16 & $1.01 \mathrm{E}-05$ & - \\
\hline 32 & $4.91 \mathrm{E}-06$ & 1.036 \\
\hline 64 & $6.74 \mathrm{E}-07$ & 2.865 \\
\hline 128 & $3.24 \mathrm{E}-08$ & 4.380 \\
\hline 256 & $1.27 \mathrm{E}-09$ & 4.670 \\
\hline
\end{tabular}

\subsection{Sedov Explosion Test}

Sedov explosion test is performed to investigate code's ability to deal with strong shocks and non-planar symmetry [3]. The problem involves a self-similar evolution of a cylindrical blastwave in a uniform grid $(N=100)$ from a localized initial pressure perturbation (delta-function) in an otherwise homogeneous medium. Governing equations are given in Eq. (15) and the fluxes are evaluated with Rusanov scheme and GKS [5]. For the code initialization, dimensionless energy $\epsilon=1$ 
is deposited into a small region of radius $\delta=3 \Delta R$. Inside this region, the dimensionless pressure $P_{0}^{\prime}$ is given by Eq. (19).

$$
P_{0}^{\prime}=\frac{3(\gamma-1) \epsilon}{(m+2) \pi \delta^{(m+1)}}
$$

where $m=1$ for cylindrical geometry. Reflecting boundary condition is employed at the center $(R=0)$, whereas boundary condition at $R=1$ is not required for this problem. The initial velocity and density inside the domain are 0 and 1 respectively and the initial pressure everywhere except the kernel is $10^{-5}$. As the source term is very stiff, the CFL number is set to be 0.1 . The final time is $t=0.05$.

Figure 3 shows that the peak for WENO-C is higher for density and is closest to the analytical value, similar to fifth-order finite difference version [3], but MUSCL has higher offset peaks for pressure and velocity. GKS performs slightly better than $\mathrm{RS}$, as the peaks are slightly higher for all the cases.
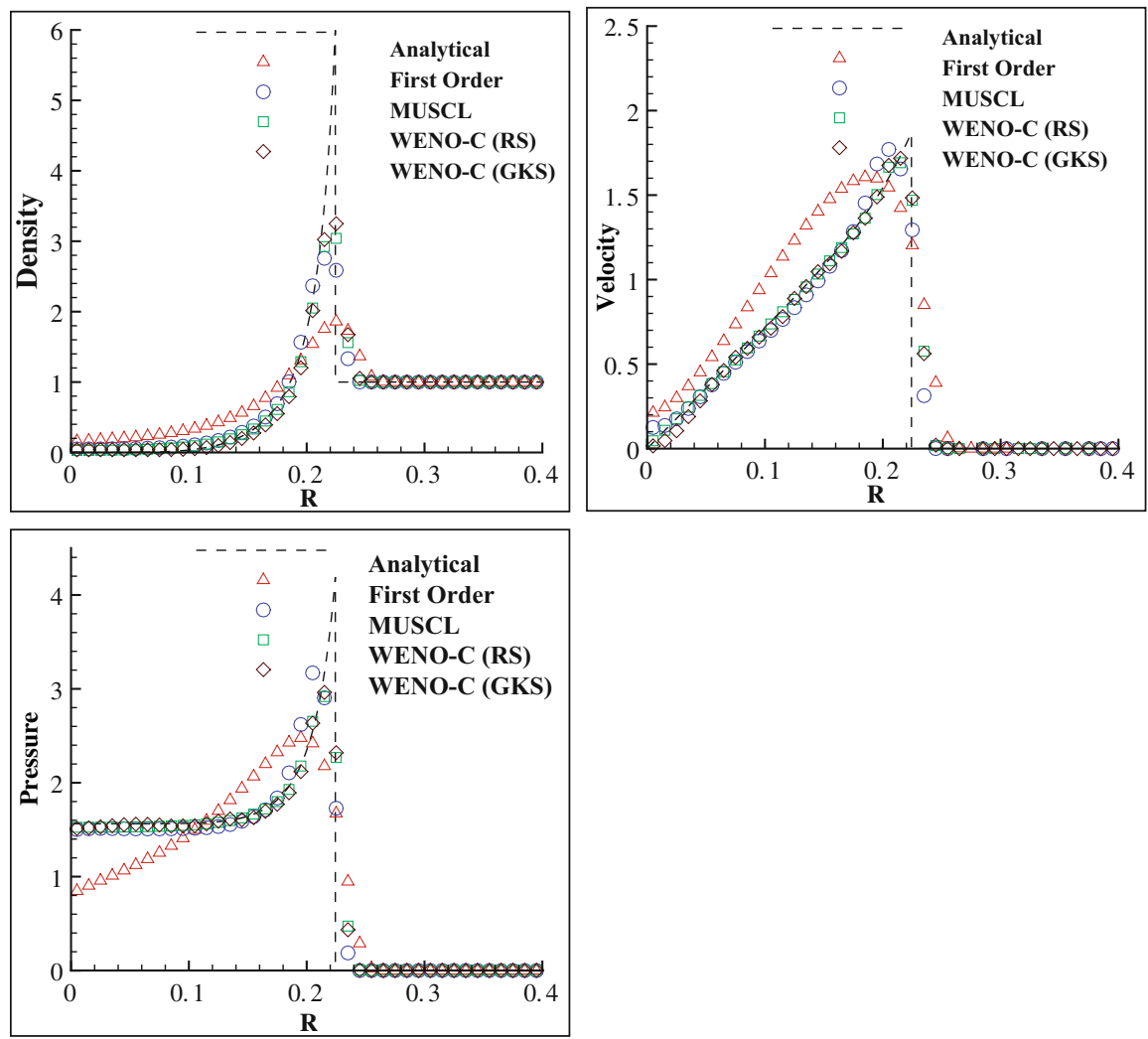

Fig. 3 Variation of density, velocity, and pressure with the radius for Sedov explosion test in cylindrical-radial coordinates. Domain is restricted to $R=0.4$ for the sake of clarity 


\subsection{Modified 2D Riemann Problem in $(R-z)$ Coordinates}

The final test for the present scheme involves a modified 2D Riemann problem in cylindrical $(R-z)$ coordinates, as illustrated in Fig. 4 (top left). The problem involves 2 contact discontinuities and 2 shocks as the initial condition, resulting in the formation of a self-similar structure propagating towards the low densitylow pressure region (region 3). The governing equations in cylindrical $(R-z)$ coordinates are provided in Eq. (20).

The computations are performed until $t=0.2$ with a CFL number of 0.5 on a domain $(R, z)=[0,1] \times[0,1]$ divided into $500 \times 500$ zones. The boundary conditions are symmetry at the center (except for the antisymmetric radial velocity) and outflow elsewhere. HLL Riemann solver is used for flux evaluations. Rich smallscale structures in the contact-contact region (region 1) can be observed from
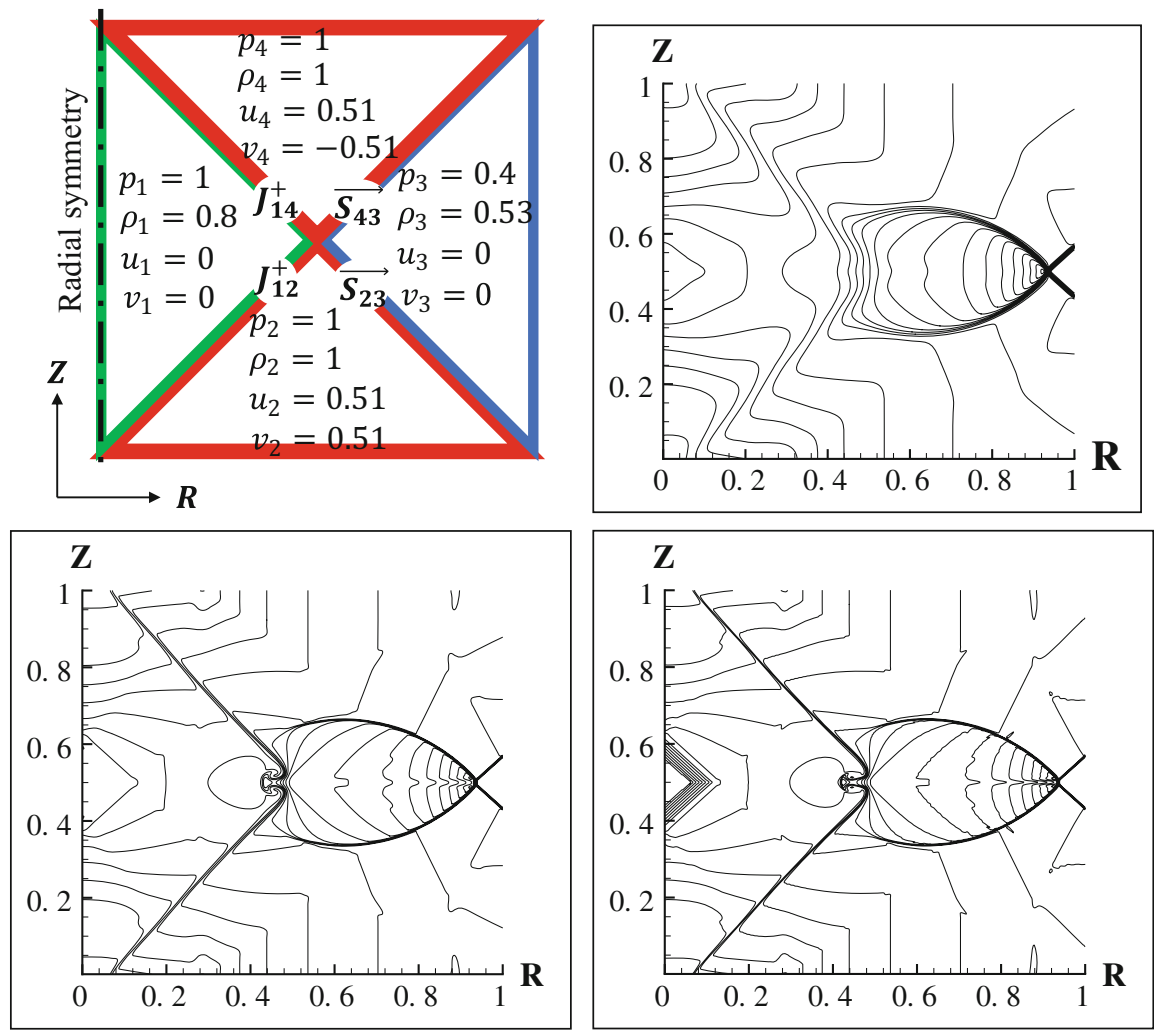

Fig. 4 Modified 2D Riemann problem in cylindrical $(r-z)$ coordinates: schematic (top left), density contours at $t=0.2$ with first-order (top right), second-order MUSCL (bottom left), and WENO-C (bottom right) reconstruction schemes 
Fig. 4 for WENO-C reconstruction, when compared with first and second-order MUSCL reconstruction. Structures are highly smeared for the case of first-order reconstruction.

$$
\frac{\partial}{\partial t}\left(\begin{array}{c}
\rho \\
\rho v_{R} \\
\rho v_{z} \\
\rho e
\end{array}\right)+\frac{1}{R} \frac{\partial}{\partial R}\left(\begin{array}{c}
\rho v_{R} R \\
\left(\rho v_{R}^{2}+p\right) R \\
\rho v_{R} v_{z} R \\
(\rho e+p) v_{R} R
\end{array}\right)+\frac{\partial}{\partial z}\left(\begin{array}{c}
\rho v_{z} \\
\rho v_{R} v_{z} \\
\rho v_{z}^{2}+p \\
(\rho e+p) v_{z}
\end{array}\right)=\left(\begin{array}{c}
0 \\
p / R \\
0 \\
0
\end{array}\right)
$$

\section{Conclusions}

The fifth-order finite-volume WENO-C reconstruction scheme is proposed for structured grids in cylindrical coordinates to achieve high order spatial accuracy along with ENO transition. A grid independent smoothness indicator is derived for this scheme. For uniform grids, the analytical values in cylindrical-radial coordinates for the limiting case $(R \rightarrow \infty)$ conform to WENO-JS. Linear stability analysis of the present scheme is performed using a scalar advection equation in radial coordinates. Several tests involving smooth and discontinuous flows are performed, which testify for the fifth-order accuracy and ENO property of the scheme.

\section{References}

1. Jiang, G.-S., Shu, C.-W.: Efficient implementation of weighted ENO schemes. J. Comput. Phys. 126(1), 202-228 (1996)

2. Mignone, A.: High-order conservative reconstruction schemes for finite volume methods in cylindrical and spherical coordinates. J. Comput. Phys. 270, 784-814 (2014)

3. Shadab, M.A., Balsara, D., Shyy, W., Xu, K.: Fifth order finite volume WENO in general orthogonally-curvilinear coordinates. Comput. Fluids. 190, 398-424 (2019)

4. Liu, H., Jiao, X.: WLS-ENO: Weighted-least-squares based essentially non-oscillatory schemes for finite volume methods on unstructured meshes. J. Comput. Phys. 314, 749-773 (2016)

5. Xu, K.: A gas-kinetic BGK scheme for the Navier-Stokes equations and its connection with artificial dissipation and Godunov method. J. Comput. Phys. 171(1), 289-335 (2001) 
Open Access This chapter is licensed under the terms of the Creative Commons Attribution 4.0 International License (http://creativecommons.org/licenses/by/4.0/), which permits use, sharing, adaptation, distribution and reproduction in any medium or format, as long as you give appropriate credit to the original author(s) and the source, provide a link to the Creative Commons licence and indicate if changes were made.

The images or other third party material in this chapter are included in the chapter's Creative Commons licence, unless indicated otherwise in a credit line to the material. If material is not included in the chapter's Creative Commons licence and your intended use is not permitted by statutory regulation or exceeds the permitted use, you will need to obtain permission directly from the copyright holder. 